\title{
Lymphangioleiomyomatosis Complicated by Gestational Trophoblastic Tumor: An Ominous Coincident
}

\author{
Soheil Peiman ${ }^{1,2}$, Besharat Rahimi ${ }^{1}$, Violet Zaker Esteghamati ${ }^{2}$, Sara Sagharnia² and Seyed Farshad Allameh ${ }^{2 *}$ \\ ${ }^{1}$ Advanced Thoracic Research Center, Tehran University of Medical Sciences, Tehran, Iran \\ ${ }^{2}$ Department of Internal Medicine, Tehran University of Medical Sciences, Tehran, Iran
}

\begin{abstract}
Lymphangioleiomyomatosis is a rare progressive cystic lung disease affecting young women in childbearing ages. It generally presents with respiratory manifestations varying from simple cough to recurrent pneumothorax and chylothorax. There is known female gender predominance, highlighting the important roles for sex hormones in the pathophysiology and progression of this disease. In this case report we describe a 26 year old woman with history of dyspnea and pneumothorax during her molar pregnancy.
\end{abstract}

\section{Introduction}

Lymphangioleiomyomatosis (LAM) is a rare (prevalence of 1 in $1,000,000)$ progressive disease of lungs and lymphatics that predominantly affects women of childbearing age. This cystic lung disease results in clinical clinical syndrome characterized by dyspnea, recurrent pneumothorax, hemoptysis and chylothorax $[1,2]$. Considering the likely role of female sex hormones in pathophysiology and prognosis of LAM, pregnancy is assumed as a worsening factor for this disease $[3,4]$.

Gestational trophoblastic disease (GTD) consists of a group of neoplastic diseases that arise from the placenta producing the beta subunit of human chorionic gonadotropin (B- hCG) that can mimic the true pregnancy [5].

In this article we report a 26 year old woman in her second pregnancy (which was complicated by GTD) with associated LAM.

\section{Case Report}

On February 2014, a 26-year-old woman was presented to emergency department of Imamkhomeini Hospital of Tehran University of Medical Sciences with pleuretic chest pain and sudden onset dyspnea. She reported 8 years history of gradually progressive dyspnea, non-productive cough and fatigue, started after her first pregnancy. She didn't seek medical care for her symptoms during these years, but she noted that the symptoms had been evident in her usual activity in the last year. Recent symptoms began to aggravate by her second pregnancy which was diagnosed after 2 months of amenorrhea. She was a non-smoker and didn't report recent trauma or taking any drugs including oral contraceptives. Her 8 year-old son was healthy without medical problem. There was no significant medical problem in her first degree relatives. On examination she was agitated with associated respiratory distress, using accessory respiratory muscles. Her vital signs were as follow: Blood Pressure: 100/60 mmHg, pulse rate: $100 / \mathrm{min}$, respiratory rate: $34 / \mathrm{min}$, oral temperature: $36.8^{\circ} \mathrm{C}$ and arterial O2sat: $90 \%$. Chest examination showed decreased breath sound in left hemithorax. All other examinations were unremarkable.

Complete blood count, blood sugar, urea, creatinine, electrolytes and liver function tests were all within normal ranges. The chest radiography showed left pneumothorax with associated diffuse cystic lesion in both lungs. Spiral thoracic computed tomography (CT) scan revealed multiple bilateral thin walled cystic lesions in various sizes with associated large left pneumothorax (Figure 1a). These finding were compatible with the diagnosis of LAM. Placement of chest tube allowed the left lung to re-expand (Figure 1b).
Echocardiography was normal and pelvic ultrasonography revealed distended uterine cavity, filled by echogenic soft tissue masses with small cystic components, most compatible with molar pregnancy. Abdominopelvic CT scan did not discover any renal mass. Her B- hCG titer was $186 \mathrm{mlu} / \mathrm{ml}$. After endometrial curettage the BhCG titer decreased to $134 \mathrm{mlu} / \mathrm{ml}$ and the pathologic report revealed trophoblastic cells without malignant cells. Subsequently, pleurodesis and diagnostic lung biopsy was recommended to the patient, but she refused and was discharged and advised to check serial $\mathrm{B}$ - hCG, liver function test and monitoring of pulmonary symptoms. One month later, on follow-up, the B- hCG titer showed a rising level $(185 \mathrm{mlu} /$ $\mathrm{ml})$. Pelvic MRI revealed an abnormal enhancing $17 \times 34 \mathrm{~mm}$ lesion, with invasion to the posterior wall of uterus (Figure 2). Hysterectomy was performed and histopathological report of the specimen noted the malignant placental site trophoblastic tumor with involvement of posterior uterine wall. She underwent chemotherapy with Actinomycin D. After the first course of chemotherapy, she experienced sudden worsening of dyspnea and chest pain. Pulmonary imaging this time showed bilateral large pneumothorax. She was hospitalized again and bilateral chest tube was inserted. She refused surgical pleurodesis

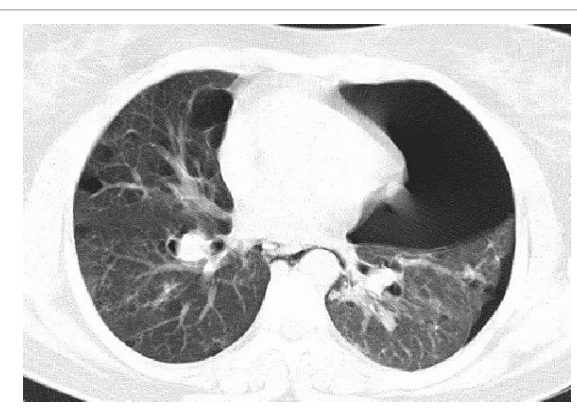

Figure 1a: Spiral thoracic computed tomography scan, showing bilateral parenchymal cystic change and associated right pneumothorax.

*Corresponding author: Seyed Farshad Allameh, Assistant Professor of internal medicine, Tehran University of Medical Sciences, Tehran, Iran, Tel: +98 21 66939922; E-mail: farshad125@yahoo.com

Received September 02, 2014; Accepted February 27, 2015; Published March 03, 2015

Citation: Peiman S, Rahimi B, Esteghamati VZ, Sagharnia S, Allameh SF (2015) Lymphangioleiomyomatosis Complicated by Gestational Trophoblastic Tumor: An Ominous Coincident. J Pulm Respir Med 5: 248. doi:10.4172/2161-105X.1000248

Copyright: @ 2015 Peiman S, et al. This is an open-access article distributed under the terms of the Creative Commons Attribution License, which permits unrestricted use, distribution, and reproduction in any medium, provided the original author and source are credited. 


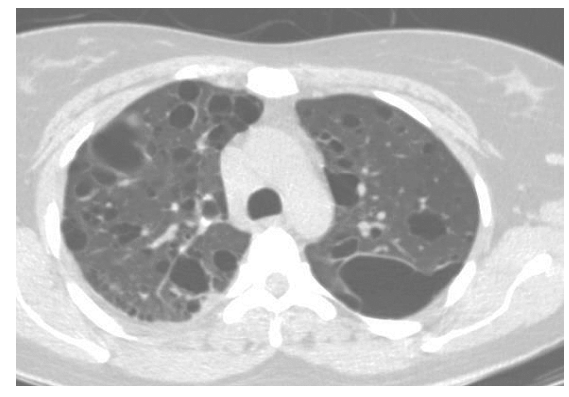

Figure 1b: High resolution computed tomography of lung after re-expansion of right lung revealing the underlying parenchymal abnormalities more precisely.

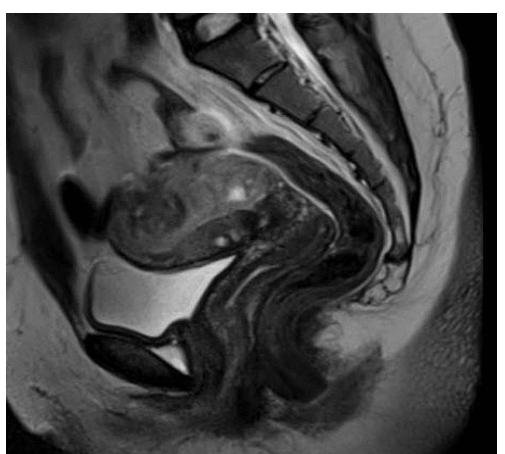

Figure 2: T2-weighted MRI of pelvis in sagittal view, showing an abnormal enhancing lesion measuring about $17 \times 34 \mathrm{~mm}$ extends from endometrial cavity to posterior wall of uterus close to serosa.

again and underwent bilateral talc pleurodesis. But 3 weeks later she was presented with right pneumothorax and pleurodesis was repeated. This patient is now in close follow-up with supportive treatments. Even though she is functional, she still has dyspnea of functional class II of New York Heart Association (NYHA).

\section{Discussion}

LAM is a progressive disease that has two forms: sporadic or those associated with tuberous sclerosis complex [1,2]. Although lung biopsy that was refused by our patient is the gold standard for diagnosis, many experts reserve it for doubtful cases [2]. Based on existing guidelines, our patient who had characteristic features on HRCT $(>10$ thin-walled round well-defined cysts with no interstitial lung disease) in addition to associated compatible clinical features (multiple and/or bilateral pneumothorax) could be categorized as Probable LAM [6]. Other diseases that cause diffuse cystic lung disease include: Pulmonary Langerhans Cell Histiocytosis, Lymphocytic Interstitial Pneumonia, Amyloidosis, Birt-Hogg-Dubé Syndrome and Cystic Pulmonary Metastatic Disease [7]. Although there are rare case reports of cystic lung disease due to metastasis [8,9], or pneumothorax as the result of metastasis [10], there are some facts in this patient that are in favor of LAM instead of metastasis as primary disease: our patient was a woman in childbearing age; she reported the onset of her symptoms from 8 years ago after her first pregnancy; her menstruation was regular till 2 months before the occurrence of pneumothorax, so the GTD has been ensued recently; in addition to characteristic cystic lung involvement, she had multiple, bilateral and recurrent pneumothorax which is common in LAM especially during gestations.

On the other hand, similar to this patient who experienced a second episode of pneumothorax after receiving chemotherapy by Actinomycin D, there are reports of pneumothorax complicating chemotherapy regimens (including Actinomycin D) for various tumors [11]. Surprisingly there is another existing report of LAM (without previous history of pneumothorax) in which initiation of chemotherapy due to associated breast cancer, leads to occurrence of recurrent pneumothorax and pneumomediastinum [12]. It seems that in these patients, initiation of the induction chemotherapy requires a special concern regarding the occurrence of pneumothorax, mandating close monitoring of respiratory system.

There are well described evidences that pregnancy or other high estrogen states could increase the risk of pneumothorax or chylothorax in LAM $[13,14]$. However GTD secrete less amount of estrogen than normal placental tissue [15]. Worsening of our patient's condition by recurrent pneumothorax despite no associated pregnancy may signify a possible pathophysiological role of human chorionic gonadotropin in LAM. This has to be confirmed by further studies.

Unfortunately there is no curative therapy and treatment of this progressive disease is still supportive which includes avoiding medications that contain estrogen, maintaining a normal weight and refraining from smoking. Other treatments such as inhaled bronchodilators or pulmonary rehabilitation may also be helpful to some extent. Apart from special clinical situations, there is no strong evidence for routine prescribing of other treatments such as Sirolimus, progesterone or other anti-estrogen interventions [6]. Lung transplantation has been recommended when symptoms reach NYHA functional class III or IV [6]. Our patient is now in NYHA functional class II and she also has associated active neoplasm, so she is not a suitable candidate for lung transplantation.

To the best of our knowledge, this is the first report of LAM, complicated with gestational trophoblastic neoplasia, making an ominous association.

\section{References}

1. McCormack FX (2008) Lymphangioleiomyomatosis: a clinical update. Chest 133: 507-516.

2. Johnson SR (2006) Lymphangioleiomyomatosis. Eur Respir J 27: 1056-1065.

3. Glassberg MK, Elliot SJ, Fritz J, Catanuto P, Potier M, et al. (2008) StetlerStevenson W, Karl M. Activation of the estrogen receptor contributes to the progression of pulmonary lymphangioleiomyomatosis via matrix metalloproteinase-induced cell invasiveness. J Clin Endocrinol Metab 93: 1625-1633.

4. Cohen MM, Freyer AM, Johnson SR (2009) Pregnancy experiences among women with lymphangioleiomyomatosis. Respir Med 103: 766-772.

5. Lurain JR (2010) Gestational trophoblastic disease I: epidemiology, pathology clinical presentation and diagnosis of gestational trophoblastic disease, and management of hydatidiform mole. Am J Obstet Gynecol 203: 531-539.

6. Johnson SR, Cordier JF, Lazor R, Cottin V, Costabel U, et al. (2010) European Respiratory Society guidelines for the diagnosis and management of lymphangioleiomyomatosis. Eur Respir J 35: 14-26.

7. Seaman DM, Meyer CA, Gilman MD, McCormack FX (2011) Diffuse cystic lung disease at high-resolution CT. AJR Am J Roentgenol 196: 1305-1311.

8. Ng CS, Wan S, Lee TW, Yim AP (2005) Cystic lesions of the lung: a forgotten menace. Eur Respir J 26: 748-749.

9. Imokawa S, Uehara M, Uto T, Sagisaka S, Sato J, et al. (2013) Pulmonary metastasis from urothelial carcinoma showing progressive multiple cystic lesions. Am J Respir Crit Care Med 188: 1267-1268.

10. Wright JD, Powell MA, Horowitz NS, Huettner PC, White F, et al. (2002) Placental site trophoblastic tumor presenting with a pneumothorax during pregnancy. Obstet Gynecol 100: 1141-1144.

11. Biran H, Dgani R, Wasserman JP, Weissberg D, Shani A (1992) Pneumothorax following induction chemotherapy in patients with lung metastases: a case report and literature review. Ann Oncol 3: 297-300. 
Citation: Peiman S, Rahimi B, Esteghamati VZ, Sagharnia S, Allameh SF (2015) Lymphangioleiomyomatosis Complicated by Gestational Trophoblastic Tumor: An Ominous Coincident. J Pulm Respir Med 5: 248. doi:10.4172/2161-105X.1000248

12. Kelly E, Mhurchu EN, Sukor S, McDonnell TJ, Tryfonopoulos D, et al. (2010) Chemotherapy-associated recurrent pneumothoraces in lymphangioleiomyomatosis. Respir Care 55: 1491-1494.

13. Fujimoto M, Ohara N, Sasaki H, Funakoshi T, Morita H, et al. (2005) Pregnancy complicated with pulmonary lymphangioleiomyomatosis: case report. Clin Exp Obstet Gynecol 32: 199-200.
14. Warren SE, Lee D, Martin V, Messink W (1993) Pulmonary lymphangiomyomatosis causing bilateral pneumothorax during pregnancy. Ann Thorac Surg 55: 998-1000.

15. Goodwin TM, Hershman JM (1997) Hyperthyroidism due to inappropriate production of human chorionic gonadotropin. Clin Obstet Gynecol 40: 32-44. 